\title{
Prevention of vascular events in diabetes mellitus: which "antithrombotic" therapy?
}

\author{
A. Ceriello, E. Motz
}

Department of Pathology and Medicine, Clinical and Experimental, Chair of Internal Medicine, University of Udine, Udine, Italy

Drs. Cimminiello and Milani present an interesting review of the literature on the efficacy of aspirin in preventing cardiovascular disease in diabetic patients. They underscore the lack of substantial evidence that diabetic patients with peripheral arterial disease (PAD) may benefit from the preventive effect of aspirin on cardiac and brain injury which has been observed in other high-risk subjects. They point out that prophylactic therapy with aspirin may be somewhat less efficacious in diabetic compared to non-diabetic patients as far as coronary events and stroke are concerned, and emphasize that in diabetic patients with PAD, aspirin does not appear to give any protection with respect to limb ischaemia.

It is true that collecting data from a population of diabetic patients without distinguishing between subgroups such as insulin-dependent and non-insulin-dependent diabetes mellitus and between patients taking insulin or not may affect the significance of the results. Moreover, any report on the prophylaxis of cardiovascular disease in diabetic patients should take into account their blood glucose and/or glycated haemoglobin levels during the follow-up period. Indeed, recent epidemiological studies have stressed the importance of different levels of metabolic control in conditioning the development of macrovascular complications in non-insulin-dependent diabetes [1-3]. Also, meta-analytic studies are hampered by the different dosages of aspirin employed in previous studies. This may be even more important in a diabetic population, in which, as recently noted by Yudkin [4], because of increased turnover of platelets higher doses of aspirin may be necessary to achieve the same levels of thromboxane inhibition as in non-diabetic patients.

Many antiplatelet agents are currently under investigation, and preliminary results are good. The

Corresponding author: Dr. A. Ceriello, Department of Pathology and Medicine, Clinical and Experimental, Chair of Internal Medicine, University of Udine, P.le S. Maria della Misericordia, I-33100 Udine, Italy

Abbreviations: PAD, Peripheral arterial disease.
GPIIb/IIIa receptor plays a crucial role in platelet aggregation and platelet thrombus formation [5]. Prospectively, the evaluation of anti-GPIIb/IIIa therapy in man may be an exciting step [5]. In the near future, very interesting results may derive from clinical trials on two compounds, ridogrel and picotamide, which combine blocking of thromboxane synthase and the thromboxane receptors. After myocardial infarction the incidence of new ischaemic events during hospital stay has been reported to be lower in patients treated with ridogrel compared to those treated with aspirin: this would be the first demonstration of a drug functioning better than aspirin in such patients [6]. Picotamide has been found to be only slightly efficacious in non-diabetic PAD patients [7], whereas in diabetic patients it prevented about $45 \%$ of expected cardiovascular events, a brilliant performance if it can be confirmed [8].

Theoretically, drugs interfering with thromboxane synthase and the thromboxane receptor should be more active than aspirin in preventing vascular complications in diabetic subjects and possibly in other groups of patients. In diabetes the level of protein glycation and oxidative stress appear to be important in the pathogenesis of macroangiopathic complications [9]. It has been demonstrated that free radicals are generated during the glycation of proteins [10] and that they increase peroxidation of arachidonic acid [11]. Moreover, free radicals have been shown to be able to directly induce, in vitro and in vivo, the formation of endoperoxides (F2-isoprostane and others), which directly bind to and interact with thromboxane receptors in addition to being a substrate for the endoperoxidase that follows cyclooxygenase in the biosynthetic pathway of thromboxane $[12,13]$. Thus, it is possible that in hyperglycaemia, a relevant quantity of thromboxane and endoperoxides which have similar actions may be generated by-passing the cyclooxygenase step. Should these alternative pathways to thromboxane-receptor stimulation have a major pathogenetic role, blocking cyclooxygenase by administration of aspirin would clearly be less useful in 
diabetic subjects, especially in the case of poor control of blood glucose levels, as well as in any other condition of elevated free-radical generation. On the contrary, the inhibition of endoperoxidase would retain part, and thromboxane receptor blockade would maintain the whole of its therapeutic efficacy. This hypothesis merits careful investigation.

Are there other reasons why the undoubtedly powerful antiplatelet action of aspirin does not render this drug more effective in preventing thrombotic events, particularly in diabetic patients? The fact is that platelet aggregation is only one of several mechanisms involved in thrombogenesis. Aspirin itself also appears to have an inhibitory effect on thrombin generation which may contribute to its effectiveness; this activity is markedly reduced in hypercholesterolaemia [14], which occurs frequently in diabetes. In diabetic as well as in non-diabetic patients with PAD, fibrinogen has been shown to be a powerful predictor of mortality $[15,16]$. It plays an important role in platelet activation [17], and high fibrinogen plasma levels in both diabetic [18] and in non-diabetic [19] subjects appear to be related to activation of thrombin generation, revealed by two markers, i.e. prothrombin fragments and D-dimer. Increased plasma levels of the latter have been reported in patients with PAD [20], as they are predictive of the risk of cardiovascular events, and in diabetic subjects [18]. In summary there is evidence to suggest that the most effective prevention of cardiovascular complications in high-risk subjects might be represented by a combination of anti-coagulant and antiplatelet drugs. Obviously this would increase costs and the incidence of undesirable side effects, and the balance between risks and benefits should be carefully assessed. Clinical trials combining oral anticoagulants and antiplatelet drugs are underway [21].

In fact, no definite conclusion can be drawn either for or against the efficacy of aspirin in any subgroup of diabetic patients with PAD. It is interesting that much the same evidence that led Drs. Cimminiello and Milani to debate the therapeutic value of aspirin in diabetes, has been cited by Yudkin supporting its administration [4]. Because of the long duration of prospective studies on this topic, we should not wait for the results of more trials on the effects of aspirin before we start collecting data on other drugs. A concerted effort should be made to define the therapeutic value of the various antiplatelet drugs as well as of anticoagulants and antioxidants, possibly in double-blind placebo-controlled trials. Data should be recorded with particular attention concerning the classification of patients at risk for cardiovascular disease, and particularly those with diabetes, into appropriate subgroups. An effort should also be made to take into account the known and newer risk markers, so that their diagnostic and prognostic value be clearly defined also as a guide to therapy.

\section{References}

1. Uusitupa MIJ, Niskanen LK, Siitonen O, Voutilainen E, Pyörälä K (1990) 5-years incidence of atherosclerotic vascular disease in relation to general risk factors, insulin levels, and abnormalities in lipoprotein composition in non-insulin-dependent diabetes and nondiabetic subjects. Circulation 82: 27-36

2. Kuusisto J, Mykkänen L, Pyörälä K, Laakso M (1994) NIDDM and its metabolic control predict coronary heart disease in elderly subjects. Diabetes 43: 960-967

3. Lehto S, Rönnemaa T, Pyörälä K, Laakso M (1996) Predictors of stroke in middle-aged patients with non-insulin dependent diabetes. Stroke 27: 63-68

4. Yudkin JS (1995) Which diabetic patients should be taking aspirin? BMJ 311: 641-642

5. Coller BS, Anderson K, Weisman HF (1995) New antiplatelet agents: platelet GPIIb/IIIa antagonists. Thromb Haemostas 74: 302-308

6. The RAPT Investigators (1994) Randomized trial of ridogrel, a combined thromboxane A2 synthase inhibitor and thromboxane A2/prostaglandin endoperoxide receptor antagonist, versus aspirin as adjunct to thrombolysis in patients with acute myocardial infarction. The Ridogrel versus Aspirin Patency Trial (RAPT). Circulation 89: 588-595

7. Balsano F, Violi F and the ADEP Group (1993) Effect of picotamide on the clinical progression of peripheral vascular disease. A double-blind placebo-controlled study. Circulation 87: 1563-1569

8. Violi F, Longoni A, Castiglioni C and the ADEP Group (1995) Effect of picotamide in preventing cardiovascular events in 438 diabetic patients: results of a multi-centre, placebo controlled, 18month study. J Am Coll Cardiol 26: 109 (Abstract)

9. Giugliano D, Ceriello A, Paolisso G (1996) Oxidative stress and diabetic vascular complications. Diabetes Care 19: 257-267

10. Ceriello A, Quatraro A, Giugliano D (1992) New insights on nonenzymatic glycosylation may lead to therapeutic approaches for the prevention of diabetic complications. Diabet Med 9: 297-299

11. Mullarkey CJ, Edelstein D, Brownlee M (1990) Free radical generation by early glycation products: a mechanism for accelerated atherogenesis in diabetes. Biochem Biophys Res Commun 173: 932-939

12. Morrow JD, Harris TM, Roberts II LJ (1990) Noncyclooxygenase oxidative formation of a series of novel prostaglandins: analytical ramifications for measurement of eicosanoids. Anal Biochem 184: $1-10$

13. Morrow JD, Hill KE, Burk RF, Nammour TM, Badr KF, Roberts II LJ (1990) A series of prostaglandin F2-like compounds are produced in vivo in humans by a non-cyclooxygenase, free radical-catalyzed mechanism. Proc Natl Acad Sci USA 87: 9383-9387

14. Szczeklik A (1995) Thrombin generation in myocardial infarction and hypercholesterolemia: effects of aspirin. Thromb Haemostas 74: $77-80$

15. Lowe GDO, Fowkes FGR, Dawes J et al. (1993) Blood viscosity, fibrinogen, and activation of coagulation and leukocytes in peripheral arterial disease and the normal population in the Edinburgh Artery Study. Circulation 87: 1915-1920

16. Ganda OP, Arkin CH (1992) Hyperfibrinogenaemia. An important risk factor for vascular complications in diabetes. Diabetes Care 15: $1245-1250$

17. Ruggeri ZM (1995) The role of von Willebrand factor and fibrinogen in the initiation of platelet adhesion to thrombogenic surfaces. Thromb Haemostas 74: 460-463

18. Ceriello A, Taboga C, Giacomello R et al. (1994) Fibrinogen plasma levels as a marker of thrombin activation in diabetes. Diabetes 43: 430-432

19. Ceriello A, Pirisi M, Giacomello R et al. (1994) Fibrinogen plasma levels as a marker of thrombin activation: new insights of the role of fibrinogen as a cardiovascular risk factor. Thromb Haemost 71: 593-595

20. Fowkes FGR, Lowe GDO, Housley E et al. (1993) Cross-linked fibrin degradation products, progression of peripheral arterial disease, and risk of coronary heart disease. Lancet 342: 84-86

21. Vermylen J (1995) Clinical trials of primary and secondary prevention of thrombosis and restenosis. Thromb Haemostas 74: 377-381 